\title{
Hiatal repair in Ivor Lewis minimally invasive esophagectomy: a case report
}

\author{
Xu-Heng Chiang, Ke-Cheng Chen, Pei-Ming Huang, Pei-Wen Yang, Mong-Wei Lin, Jang-Ming Lee \\ Department of Surgery, National Taiwan University Hospital and National Taiwan University College of Medicine, Taipei \\ Correspondence to: Jang-Ming Lee, MD, PhD. Department of Surgery, National Taiwan University Hospital and National Taiwan University College \\ of Medicine, No.7, Chung-Shan South Rd., Taipei. Email: jangminglee@gmail.com.
}

\begin{abstract}
Hiatal hernia after minimally invasive esophagectomy (MIE) is an important postoperative complication that can cause severe morbidity or even mortality among patients with esophageal cancer. Usually repair of the hiatus immediately after esophageal reconstruction is suggested to prevent such complication. However, during the Ivor Lewis MIE procedure, hiatal repair is difficult to perform due to the deep-seated location of the hiatus after the final step of esophageal reconstruction in the right chest. The current study demonstrates a method of repairing the hiatus in the Ivor Lewis MIE. This is especially useful in the resection of a massive tumor near the esophagogastric (E-G) junction, which harbors a high risk of hiatal hernia when the hiatus is left unrepaired after extensive hiatal dissection.
\end{abstract}

Keywords: Minimally invasive esophagectomy (MIE); Ivor Lewis; McKeown; hiatal hernia; case report

Received: 23 October 2019; Accepted: 18 November 2019; Published: 05 April 2020.

doi: 10.21037/jovs.2019.11.10

View this article at: http://dx.doi.org/10.21037/jovs.2019.11.10

\section{Introduction}

Esophagectomy with reconstruction for esophageal cancer is a complex procedure with a risk of potentially serious postoperative morbidity. One of these morbidities is hiatal hernia, which may result in mortality due to bowel incarceration. Post-esophagectomy hiatal hernia has been reported in the literature $(1,2)$ with an incidence estimated to be between $0.7 \%$ and $15 \%$ (1). The incidence rate differs across studies according to the surgical methods, follow-up protocols and image diagnostic criteria used, among other factors.

During the gastric conduit preparation and esophageal reconstruction in the abdominal phase of the procedure, the hiatus is usually widened to allow gastric tube elevation through the hiatus into the mediastinum and prevent conduit obstruction. The risk of hiatal hernia can be further enhanced after completion of the laparoscopic procedure, which results in diminished postoperative tissue adhesion. In the McKeown procedure, we typically repair the widened hiatus with laparoscopic suture. However, it is difficult to repair a widened hiatus in the Ivor Lewis procedure. The first reason for this is that we cannot suture the crus muscle because the gastric conduit has not yet been pulled into the mediastinum. The second reason is that it is difficult to repair the crus muscle with thoracoscopy due to the very deep position of the hiatus and poor exposure. Thus, the crus muscle is ordinarily not repaired in our general practice with the Ivor Lewis procedure. In our own experience, there is a higher incidence of post-esophagectomy hiatal hernia in the Ivor Lewis procedure than in the McKeown procedure.

In this report, we share a surgical technique to repair the widened hiatus in the Ivor Lewis procedure.

\section{Patient selection}

We followed the NCCN (National Comprehensive Cancer Network) guideline to treat esophageal cancer. Neoadjuvant concurrent chemoradiotherapy (CCRT) was administered to patients with clinically positive lymph nodes and followed by esophagectomy. All procedures performed in this study were in accordance with the Helsinki Declaration (as revised in 2013). Written informed consent was obtained from the 


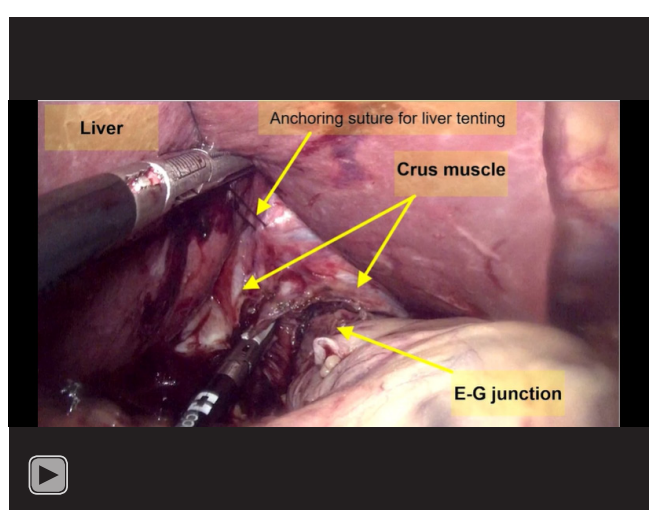

Video 1 The attached video illustrates the complete procedure step by step (4).

patient for publication of this report and any accompanying images.

Minimally invasive esophagectomy (MIE) has been our preferred method of esophagectomy since 2011, either transhiatal esophagectomy, the McKeown procedure, or the Ivor Lewis procedure. Transhiatal esophagectomy is currently excluded from our clinical practice because of the difficulty of performing mediastinal lymph node dissection. For patients with upper or middle third esophageal cancer, the McKeown procedure is suitable for them. Since the twostage Ivor Lewis procedure was first introduced in 1946, it has been the operation of choice for esophageal cancer involving the lower-third esophagus or esophagogastric (E-G) junction (3). In our hospital, we generally perform total thoracoscopic and laparoscopic Ivor Lewis esophagectomy. Sometimes we have even tried singleportal esophagectomy, gastric conduit mobilization and intrathoracic anastomosis with circular stapler.

The case we present in this report is a 34-year-old male who had no underlying disease. He presented with dysphagia and worsening of the symptoms progressed rapidly. The imaging study showed an E-G junction tumor approximately $5 \mathrm{~cm}$ in maximal diameter. Endoscopic ultrasound also revealed a tumor above the E-G junction that had almost completely obstructed the esophageal lumen. Adenocarcinoma was reported in the pathological examination. Positron emission tomography showed no distant metastasis. No adjacent organ invasion was noted in bronchoscopy and computed tomography scan. This patient was diagnosed with cT3N1M0, stage III esophageal adenocarcinoma.

\section{Pre-operative preparation}

After the patient received neoadjuvant CCRT, the tumor shrank and the post-CCRT clinical stage was diagnosed as ycT3N0M0. An Ivor Lewis procedure was performed one month after completion of neoadjuvant CCRT.

The patient was placed under intubated general anesthesia. The anesthesiologist used a single-lumen endotracheal tube with an enclosed bronchial blocker to establish one-lung ventilation for prevention of lung hindrance to the surgical field. The MIE Ivor Lewis procedure consisted of two stages; the abdominal stage followed by the thoracic stage. In the abdominal stage, the patient was set in the supine position with legs together; in the thoracic stage, the patient was set in the standard left lateral decubitus position with oblique stretching.

Equipment preference card:

(I) Abdominal wound protector: GelPOINT Advanced Access Platform (Applied Medical, California, USA);

(II) Thoracic wound protector: Alexis ${ }^{\circledR} \mathrm{O}$ Wound Protector-Retractor (Applied Medical, California, USA);

(III) Energy device: LigaSure ${ }^{\mathrm{TM}}$ Maryland (Medtronic, Minnesota, USA);

(IV) Suture device: Endo Stitch ${ }^{\mathrm{TM}}$ Suturing Device (Medtronic, Minnesota, USA);

(V) Anastomosis device: $21 \mathrm{~mm}$ DST EEA ${ }^{\mathrm{TM}} \mathrm{OrVil}^{\mathrm{TM}}$ (Medtronic, Minnesota, USA);

(VI) Suture string: V-Loc ${ }^{\mathrm{TM}}$ 180, V-20 (Medtronic, Minnesota, USA).

\section{Surgical technique (Video 1)}

\section{Abdominal stage}

A single periumbilical incision was made, and a wound protector was applied. We performed liver tenting to secure a better surgical field (5). We then divided the gastrocolic ligament and short gastric vessels and adequately exposed the crus muscle. We separated the attachments between the stomach and pancreas, then raised the stomach so that the left gastric artery and vein could be divided. The lymph nodes along the left gastric artery (station 7, Japan Esophageal Society) were dissected and removed. We used a linear stapler to form a gastric conduit. A feeding jejunostomy was also performed on the jejunum $40 \mathrm{~cm}$ from the Treitz ligament for postoperative nutrition support $(6,7)$. 


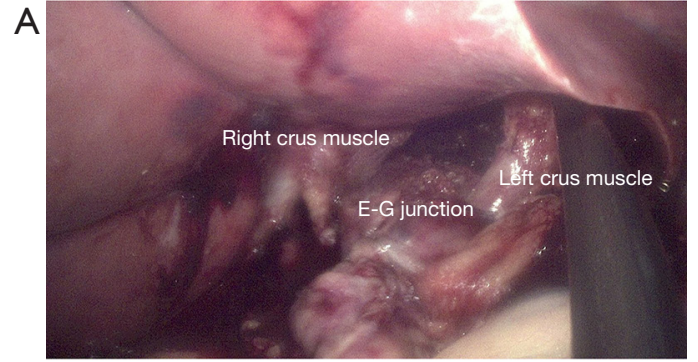

B
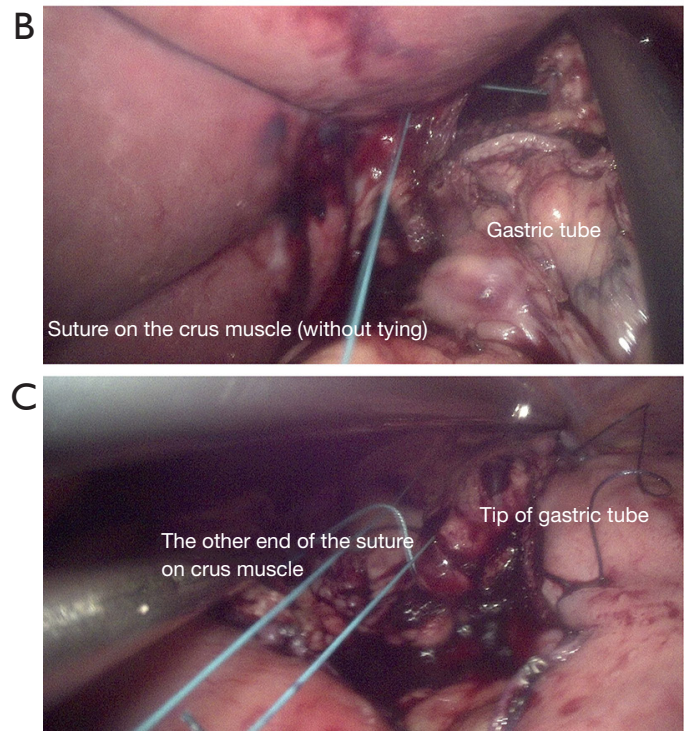

Figure 1 The abdominal stage of Ivor Lewis procedure. (A) The hiatus was widened for gastric conduit elevation into the thoracic cavity; (B) the crus muscle was sutured with Endo-stitch string (without tying); (C) the other end of Endo-stitch was anchored on the tip of the gastric conduit. E-G, esophagogastric.

Finally, we widened the hiatus to let the gastric tube pass into the mediastinum without resistance (Figure 1A). Then we sutured the crus muscle with the Endo Stitch Suturing Device without a tie (Figure 1B). We retained approximately $40 \mathrm{~cm}$ of the suture string and made an anchoring suture on the tip of the gastric conduit (Figure 1C) This allowed us to reach the string sutured on the crus muscle when we pulled the gastric conduit into the thoracic cavity in the next stage.

\section{Thoracic stage}

After completion of the first stage, we set the patient in the left decubitus position. A single thoracoscopic working port was made over the 7 th intercostal space along the middle axillary line. For better surgical exposure, the azygos vein was sacrificed and several retention stitches were anchored
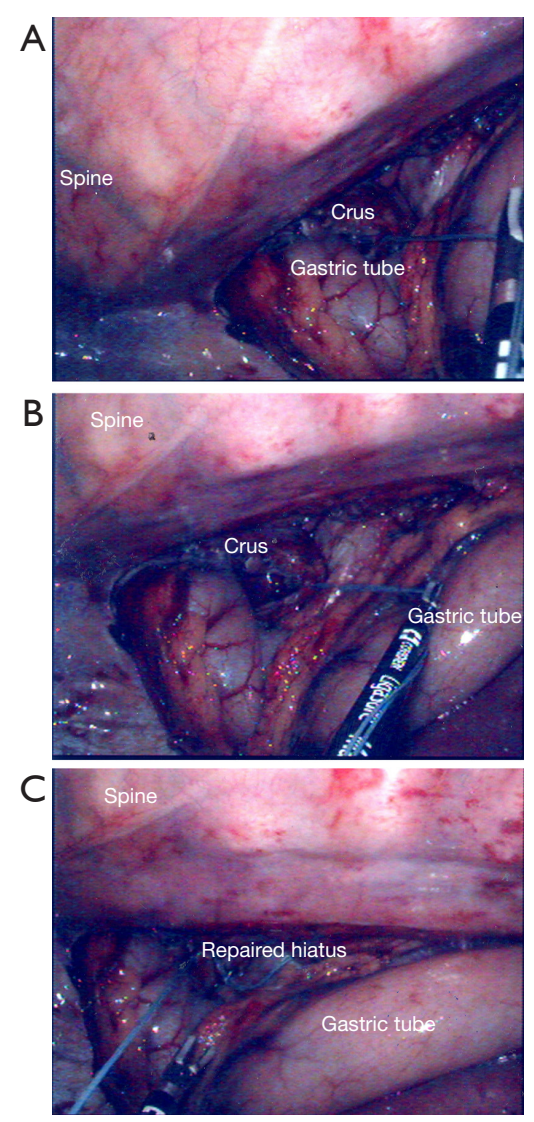

Figure 2 The thoracic stage of Ivor-Lewis procedure. (A) The previous suture was pulled into thoracic cavity along with gastric conduit; (B) the widened hiatus was exposed clearly by stretching the suture string; $(\mathrm{C})$ repair of the hiatus has been completed.

along the border of the esophagus (5). Then we dissected the esophagus extensively through the crevice to the lower mediastinum. Mediastinal lymph nodes were also dissected (station 105, 106 and 107, Japan Esophageal Society). The esophagus was divided at the level of the azygos vein (about $7 \mathrm{~cm}$ proximal to the tumor) with a $60 \mathrm{~mm}$ linear stapler. We pulled the stomach and the gastric conduit into the thoracic cavity so that the previously sutured string on the crus could be reached (Figure 2A). We performed anastomosis between the remaining esophageal stump and the gastric conduit with a $21 \mathrm{~mm}$ circular stapler. A running barbed $\mathrm{V}$-loc suture was performed for anastomosis reinforcement.

Once we reached the suture string that we sutured on the crus muscle in the abdominal stage, we were able to expose the hiatal defect clearly by stretching the string (Figure 2B). 
With good exposure, we made another suture on the crus muscle for reinforcement and tied it with a node pusher (Figure 2C).

\section{Role of the team members}

Like other surgeries, MIE requires close, cooperative teamwork to facilitate efficiency and safety in the operating room.

The surgical team consisted of:

(I) Attending surgeon: Jang-Ming Lee, MD, PhD;

(II) Assistant surgeon: Xu-Heng Chiang, MD;

(III) Anesthesiologist and nurse anesthetist;

(IV) Scrub nurse and circulating nurse.

The attending surgeon should be the leader and instruct other team members to secure good communication and coordination during the surgery.

The anesthesiologist controls cardiopulmonary stability during the operation.

\section{Postoperative management}

The patient was extubated in the operating room and then transferred to the surgical intensive care unit for post-operative care. On postoperative day two, he was transferred to the normal surgical ward. Nutrition was supported via jejunostomy feeding tube, and the patient was able to drink water perorally. The patient was discharged from the hospital on postoperative day nine. There was no surgical complication or other adverse event during the hospital stay. There was no hiatal hernia on the chest plain film 3 months after operation.

\section{Discussion and conclusions}

The introduction of MIE has significantly improved the outcome of esophagectomy, with a decrease in blood loss, respiratory distress, and postoperative pain, as well as shorter hospital stay compared to conventional open esophagectomy $(7,8)$. However, incidence of post-MIE hiatal hernia is reported to be higher than in traditional esophagectomy ( $4.5 \%$ vs. $1.0 \%$ ) (9). Thus, to prevent hiatal hernia has become an important issue with the prevalence of MIE. Especially for the Ivor Lewis procedure, effective repair of the crus muscle was highly problematic in the past. Although single portal MIE for the Ivor Lewis procedure was effective and safe in preliminary reports, there was no adequate technique to reduce frequency of postoperative hiatal hernia (10). With similar principles and procedures, it is also feasible to repair hiatal defects in the Ivor Lewis procedures performed by multi-portal MIE or open esophagectomy.

\section{Acknowledgments}

Funding: None.

\section{Footnote}

Conflicts of Interest: All authors have completed the ICMJE uniform disclosure form (available at https://jovs. amegroups.com/article/view/10.21037/jovs.2019.11.10/ coif). JML serves as an unpaid editorial board member of Fournal of Visualized Surgery from June 2019 to May 2021. The other authors have no conflicts of interest to declare.

Ethical Statement: The authors are accountable for all aspects of the work in ensuring that questions related to the accuracy or integrity of any part of the work are appropriately investigated and resolved. All procedures performed in this study were in accordance with the Helsinki Declaration (as revised in 2013). Written informed consent was obtained from the patient for publication of this report and any accompanying images. A copy of the written consent is available for review by the Editor-inChief of this journal.

Open Access Statement: This is an Open Access article distributed in accordance with the Creative Commons Attribution-NonCommercial-NoDerivs 4.0 International License (CC BY-NC-ND 4.0), which permits the noncommercial replication and distribution of the article with the strict proviso that no changes or edits are made and the original work is properly cited (including links to both the formal publication through the relevant DOI and the license). See: https://creativecommons.org/licenses/by-nc-nd/4.0/.

\section{References}

1. Price TN, Allen MS, Nichols FC, 3rd, et al. Hiatal hernia after esophagectomy: analysis of 2,182 esophagectomies from a single institution. Ann Thorac Surg 2011;92:2041-5.

2. Willer BL, Worrell SG, Fitzgibbons RJ Jr, et al. Incidence of diaphragmatic hernias following minimally invasive versus open transthoracic Ivor Lewis McKeown 
esophagectomy. Hernia 2012;16:185-90.

3. Xu L, Li JH, Ye JM, et al. A Retrospective Survival Analysis of Anatomic and Prognostic Stage Group Based on the American Joint Committee on Cancer 8th Edition Cancer Staging Manual in Luminal B Human Epidermal Growth Factor Receptor 2-negative Breast Cancer. Chin Med J (Engl) 2017;130:1945-52.

4. Chiang XH, Chen KC, Huang PM, et al. The attached video illustrates the complete procedure step by step. 2020;7:043 . Available online: http://www.asvide.com/watch/33083

5. Chuang JH, Yang SM, Yang PW, et al. Single-port minimally invasive esophagectomy. J Vis Surg 2018;4:244.

6. Yang SM, Hsiao WL, Lin JH, et al. Laparoscopic percutaneous jejunostomy with intracorporeal V-Loc jejunopexy in esophageal cancer. Surg Endosc 2017;31:2678-86.

doi: $10.21037 /$ jovs.2019.11.10

Cite this article as: Chiang $\mathrm{XH}$, Chen $\mathrm{KC}$, Huang PM, Yang PW, Lin MW, Lee JM. Hiatal repair in Ivor Lewis minimally invasive esophagectomy: a case report. J Vis Surg 2020;6:22.
7. Biere SS, van Berge Henegouwen MI, Maas KW, et al. Minimally invasive versus open oesophagectomy for patients with oesophageal cancer: a multicentre, open-label, randomised controlled trial. Lancet 2012;379:1887-92.

8. Smithers BM, Gotley DC, Martin I, et al. Comparison of the outcomes between open and minimally invasive esophagectomy. Ann Surg 2007;245:232-40.

9. Oor JE, Wiezer MJ, Hazebroek EJ. Hiatal Hernia After Open versus Minimally Invasive Esophagectomy: A Systematic Review and Meta-analysis. Ann Surg Oncol 2016;23:2690-8.

10. Lee JM, Yang SM, Yang PW, et al. Single-incision laparothoracoscopic minimally invasive oesophagectomy to treat oesophageal cancerdagger. Eur J Cardiothorac Surg 2016;49 Suppl 1:i59-63. 